\title{
In-Door Surveillance Module Based on an Associative Memory
}

\author{
Ghassan A. Mubarak \\ gasanaliraqi250@gmail.com \\ Emad I. Abdul Kareem \\ mmimad72@yahoo.com \\ Department of Computer Science, \\ College of Education, \\ A1- Mustansiriya University, Baghdad, Iraq
}

Received on: 22/09/2019

Accepted on: 19/11/2019

\begin{abstract}
Most recent studies have focused on using modern intelligent techniques especially those developed In-Door surveillance systems. Such techniques have been built depending on modern Artificial intelligence-based modules. Those modules act like a human brain, they learn and recognize what they learned. The importance of developing such systems came after the requests of customers and establishments to defend their properties and avoid Intruders' damages. This would be provided by an intelligent module that ensure the correct alarm for correct non-secured state, Thus, an Indoor surveillance module depending on Multi-Connect Architecture Associative Memory (MMCA) has been proposed. This proposed system can be trained for more than to shoot. Thus the module can recognize more than one true state that might be secured or non-secured states in real-time. The current study found an accepted accuracy level between $(62.778 .8 \%)$ at first training cycle with two images. While the final result were between $(97-100 \%)$ at the fifth training cycle with (10) images. It considered a high performance and very excellent results.
\end{abstract}

Keywords: Associative memory, MMCA Associative Memory, In-Door Surveillance System, MMCA Associative Memory Based In-Door Surveillance Module.

\section{Introduction:}

Many researchers have competed to improve the capabilities of surveillance system by adding modern artificial techniques. The purpose of this competition was the incremental demands on high-performance systems to reduce false alarm and give a certain true alert in non-secured cases. One of these systems was the In-Door surveillance system, where some researchers had developed many traditional methods and modules that had been faced with some limitations. They rather need a large storage size or had a high cost. Instead of traditional techniques, an Artificial Intelligence based Module has been developed to overcome the limitations of both storage size and $\operatorname{cost}[1]$.

It is important to review previous researches that dealt with this subject. Such as Akash, et al. (2015), it proposed a camouflage process in a dynamic environment with one clue to obtain less costly results, which had failed to control an important place [2]. Where Valery, et al. (2006), had determined internal 32 cameras control systems and fingerprint as sensors, an additional infrared identification system had been used in its proposed system design. This system identifies staff and time. It depends on NonArtificial intelligence methods [3]. 


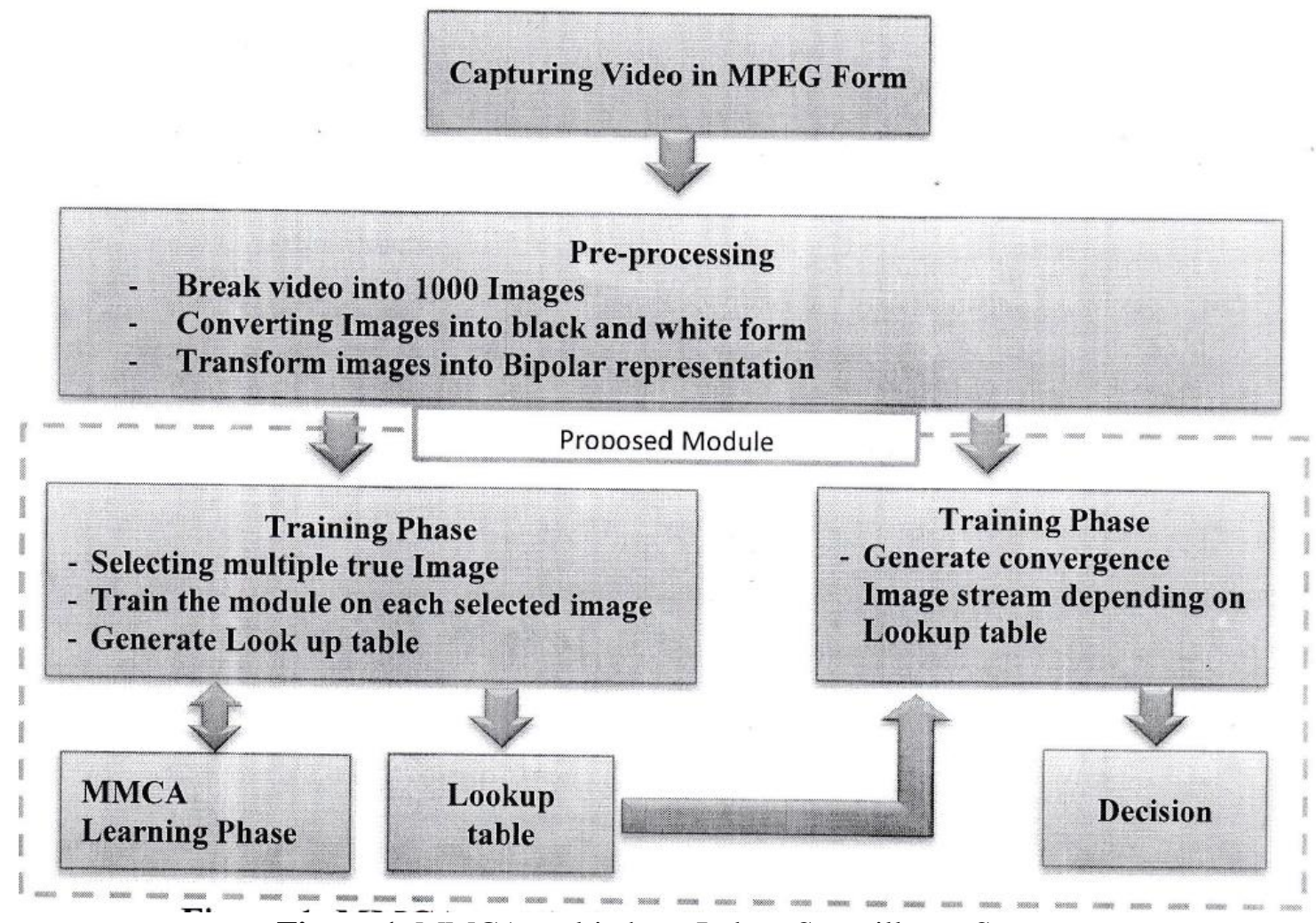

Figure 1. MMCA multi-shoot Indoor Surveillance System

Most recently invented Modified Multi connect Architecture Associative Memory (MMCA) methods has been chosen to carry out high performance with more accurate results[5] [6] [7],

The proposed Module as an MMCA has two major phases, which are: the training and the analyzing phases. This shows module capability of training on true images that might be either secured or non-secured cases. MMCA Learning phase been depended on designing of proposed module training phase. As well as the analyzing phase depends on MMCA Convergence phase (See Figure 1). The main study goal is enhancement of the indoor surveillance system by implementing. MMCA associative memory. Thus, the process of complexity has been reduced. The current proposed module would be trained on multiple true images in several training cycles. Where true images represents either secured or non-secured cases. After comparing the selected images with its given image stream, the module would be able to decide whether it is secured or non-secured image stream.

\section{Intelligent surveillance Module and system}

An In-Door surveillance module becomes of high demand that may overcome many of the security problems and crimes. Establishments, organizations, and companies rather had a fear of losing important properties or leakage of important information[4]. Security problems have extreme importance to requests more accurate systems. Thus, many projects had been made that used traditional techniques but problems still faced society [1]. A brand new In-Door surveillance system is needed, such as smart observation technology based on MMCA, which will provide a secure environment and prevent hackers [8]. An intelligent surveillance system has been developed. They depend on the neural networks which mimic the human mind has been implemented[5]. The intelligent surveillance systems in a dynamic environment push 
toward decrement of false alarms[1] [7]. In this research, an MMCA associative has been used to develop an intelligent surveillance module.

\section{Associative Memory}

The neural networks has come after many trials to simulate human brain behavior. The information has been gathered and stored in a complex associated addresses form. It stored as a set of data and weights. Initially, the Training process must be performed with determined inputs to train module on selected images. This would reduce critical images that recalled [5] the repetitive networks stores a large number of models. But still had holes that may be generated during stable cases. Therefore, An independent approximation could differ from the closest state [5] (See Figure 2).

$\mathrm{V}=[\mathrm{V} 1, \mathrm{~V} 2, \mathrm{~V} 3 \ldots, \mathrm{Vn}]$

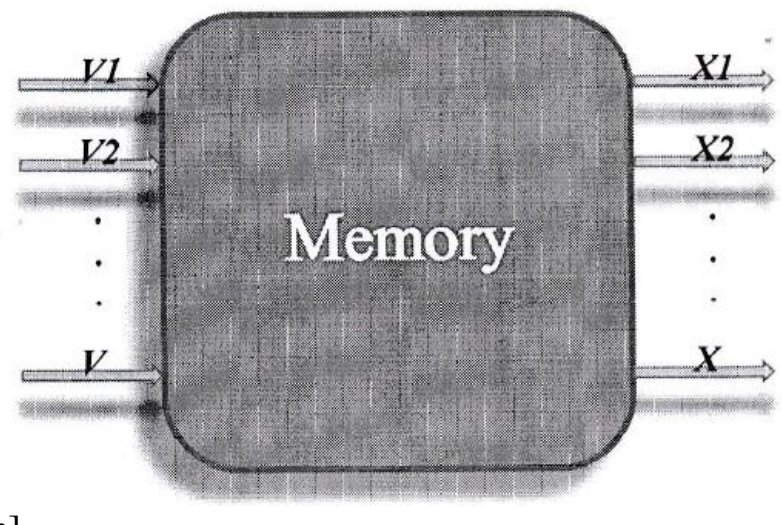

$\mathrm{X}=[\mathrm{X} 1, \mathrm{X} 2, \mathrm{X} 3 \ldots, \mathrm{Xn}]$

Figure 2. Block diagram of an associative memory [5].

The Figure 2 shows an input $\mathrm{X}$ and an output vector $\mathrm{V}$ of associative memory technique as shown in Equation (1).

Where

$$
V=M[X]
$$

$\mathrm{X}$ vector map,

$\mathrm{Z}$ specifies the vectors,

$\mathrm{M}$ is a generic operator and the expression of the matrix is non-line

This matrix shows different results. The models were selected for each type of memory inaccurate manner. In dynamic memory, the variable $M$ is linked to a time variable [5]. The input and the output area have varied. Figure illustrates Heteroassociation response as shown in bellow

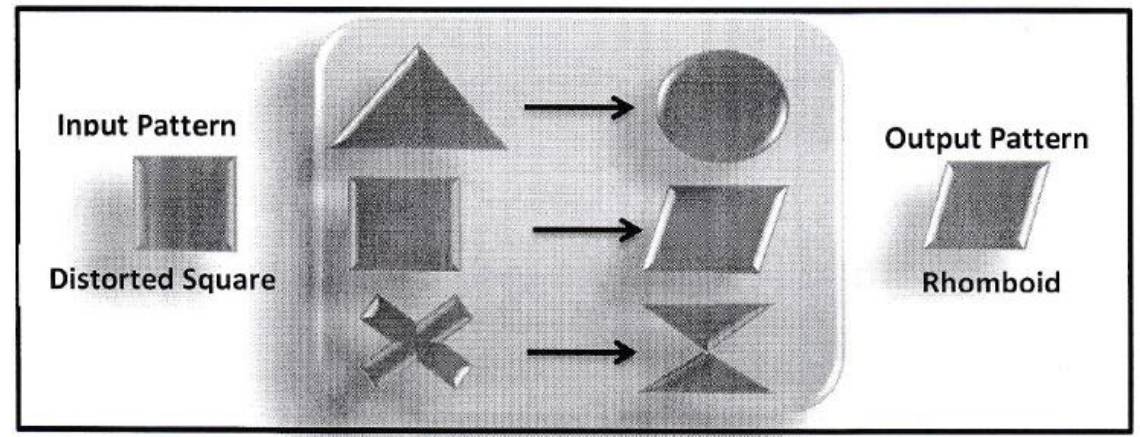

Figure 3. Hetero-association response [4] [5]. 


\section{Modify Multi Connect Architecture MMCA}

The Modify Multi Connect Architecture (MMCA) algorithm, basically consists of two essential phases. They are: the learning phase and the convergence phase. The MMCA easier and less complex than MCA where it has a comparison between pair of images according to energy function. This method would be adopted to build an algorithm for the proposed In-Door surveillance modul. MMCA fixed size is an advantage during both learning and convergence. It relies on the opposite value of images, local minimum limits, and low noise rates. MMCA would be more effective in obtaining efficiency, real-time and accuracy in the results [7] [9].

Algorithm 1 is designed to implement MMCA learning phase[7]:

\section{Algorithm 1: MMCA learning phase}

Input: training patterns $p$

Output: lookup table for all $n$ corresponding stored patterns.

Step_1: Initialize the two connection weights matrices.

$$
w_{0}=\left[\begin{array}{ll}
1 & 1 \\
1 & 1
\end{array}\right], \quad w_{1}=\left[\begin{array}{cc}
1 & -1 \\
-1 & 1
\end{array}\right]
$$

Step_2: Repeat steps 2.1 and 2.2 to the end of training pattern $p$ :

Step_2.l: Divide the training pattern $p$ to $n$ vectors with length two.

Step-2.2: For each vector $v$, repeat steps 2.2.1, 2.2.2 and 2.2.3:

Step_2.2.1: Assign the save majority description smd as follows:

$$
\begin{gathered}
\operatorname{md}(v)= \begin{cases}v[1] & v[1]<>v[2] \\
\sum_{i=1}^{2} v i & \text { otherwise }\end{cases} \\
\operatorname{smd}(v)=\text { hard limiter }(\operatorname{md}(v))=\left\{\begin{array}{cc}
1 & \operatorname{md}(v) \geq 0 \\
-1 & \operatorname{md}(v)<0
\end{array}\right.
\end{gathered}
$$

Step_2.2.2: Assign the save vector's weight svw as follow:

$$
\operatorname{svw}=f(\operatorname{Decode}(v))=\left\{\begin{array}{llllll}
0 & \text { or } & 3 & 0 & \text { \{means } & w 0 \\
1 & \text { or } & 2 & 1 & \text { \{means } & w 1
\end{array}\right.
$$

Where: Decode is a function to convert the binary number to decimal number.

End

Step_2.2.3: Save smd and syw for this vector in the lookup table.

While algorithm 2 designed for MMCA convergence phase[7]:

\section{Algorithm 2: MMCA convergence phase}

Input: $n$ of unknown patterns $p$

Output: Convergence pattern $C P$.

Step_1: Initiatize the two connection weights matrices.

Step_2: Initialize the energy function matrix $e$ :

$$
w_{0}=\left[\begin{array}{ll}
1 & 1 \\
1 & 1
\end{array}\right], \quad w_{1}=\left[\begin{array}{cc}
1 & -1 \\
-1 & 1
\end{array}\right]
$$

$$
e=\left[\begin{array}{cc}
-2 & 0 \\
0 & -2
\end{array}\right]
$$

Step_3: Repeat steps 3.1, 3.2 and 3.3 until the unknown pattern $p$ is ended: 
Step_3.l: Divide the unknown pattern $\boldsymbol{p}$ to $\boldsymbol{n}$ vectors with length two.

Step_3.2: For each vector $v$, repeat steps 3.2.1 and 3.2.2:

Step_3.2.1: Assign the test majority description tmd as follows:

$$
\begin{gathered}
\operatorname{md}(v)= \begin{cases}v[1] & v[1]<>v[2] \\
\sum_{i=1}^{2} v i & \text { otherwise }\end{cases} \\
\operatorname{tmd}(v)=\text { hard limiter }(\operatorname{md}(v))=\left\{\begin{array}{cc}
1 & \operatorname{md}(v) \geq 0 \\
-1 & \text { otherwise }
\end{array}\right.
\end{gathered}
$$

Step_3.2.2: Assign the test vector's weight tvw as follow:

$$
t v w=f(\operatorname{Decode}(v))=\left\{\begin{array}{llllll}
0 & \text { or } & 3 & 0 & \text { \{means } & w 0 \\
1 & \text { or } & 2 & 1 & \text { \{means } & w 1
\end{array}\right.
$$

Step_3.3: Sum up the energy function for al1 $n$ vectors in the unknown pattern each with its corresponding vector in the stored patterns:

$$
e p=\sum_{i=1}^{n} e\left[s v w_{i}, t v w_{i}\right] *(s m d * t m d)
$$

Step_4: Determine the stored pattern number minp with the minimum energy function to converge the unknown pattern towards it:

$$
\min p=\min (e p)
$$

Where the min function is to determine the minimum energy function in $e p$ affay.

Step_5: Repeat steps 5.1, 5.2 and 5.3 to build the final converge pattern $c p$ :

Step_5.l: Assign tempcv for each $n$ vector in the test unknown pattern $p$ :

$$
\text { tempcv } i=v_{i} \times s v w_{\min }
$$

Step_5.2: Assign the result majority description $(r m d)$ to each $n$

\section{tempcv vector.}

$$
\operatorname{md}\left(\text { tempcv }_{i}\right)=\left\{\begin{array}{cc}
v[1] & v[1]<>v[2] \\
\sum_{j=1}^{n} \text { tempcv }^{i} & \text { otherwise }
\end{array}\right.
$$

Step_5.3: Create the converge vector $c v$ in the converge pattern $c p$ :

\section{End}

$$
c r_{i}=(\text { smd } *, n, x \text { rmd }) \text { tempcv, }
$$

\section{The Proposed In-Door Surveillance Module}

Without using traditional surveillance Modules, the proposed module based on modern artificial intelligent technique that used a neural network. It is Modified MultiConnect Architecture Associative Memory (MMCA). It simulates human in learning and making decisions.

A traditional surveillance system had been made of the processing unit and cameras [10]. The cameras capture videos. The processing unit stores and analyzes it. Using MMCA associative memory enhances the performance and vision of the proposed module as an advantage. At the beginning the module would be trained on one secured image of the surveillance environment then on both secured and non-secured images to make a comparison between results for each case. The aspects of the proposed module would be shown as below. 


\subsection{High Sensitivity}

Captured videos have been pre-processed to allow manipulation with them. Thus, any given video has to be converted into black and white images, then transformed into bipolar representation. They would be suitable for the MMCA Associative Memory based module.

At first training cycle, the proposed module selected just one secured image for training. This selected image would be compared to the later captured video in the same place. But in the later cycles, more true training images have been selected. The module would be more accurate in recognizing any unsafe events that could occur in video capture duration. As mentioned before, the bi-polar form gives more sensitivity at training and analyzing phases. It minimizes the false cases that might be happening.

\subsection{Low Storage size}

The proposed module depends on MMCA which is a single layer neural network. Thus, it has a constant size whatever dealing a large amount of data that consists of frames. Each node represents a vector of frame. The purpose of that it just has two neural nodes (See: figure 4) [3].

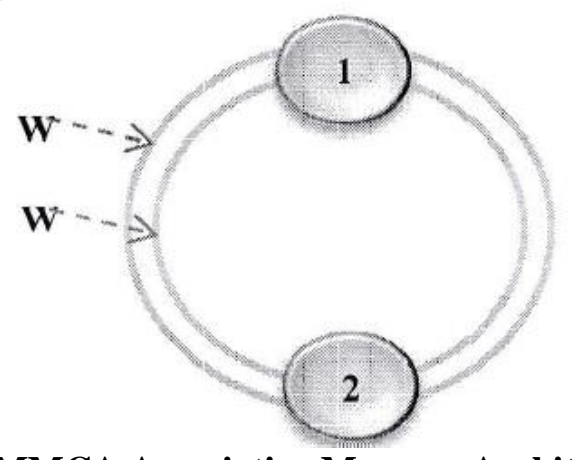

Figure 4. MMCA Associative Memory Architecture [3].

The above figure illustrates both of two neural nodes" They are connected by two connections. They all produced a small neural network which has just two weights w0 and $\mathrm{w} 1$. The network's small size made the proposed module more efficient [4].

The proposed module reduced the number of vectors by eliminating zeros of diagonal from training pre-processed image. Thus, two weights were used to build convergence image stream [5]. The proposed module weights would be as shown in table 1 .

Table 1: States of weights of MMCA

\begin{tabular}{|c|c|c|}
\hline $\begin{array}{c}\text { Binary representation } \\
\text { of vectors }\end{array}$ & $\begin{array}{c}\text { vector } \\
\text { state }\end{array}$ & Weights \\
\hline 00 & $-1-1$ & {$\left[\begin{array}{ll}1 & 1 \\
1 & 1\end{array}\right]$} \\
\hline 01 & -11 & {$\left[\begin{array}{cc}1 & -1 \\
-1 & 1\end{array}\right]$} \\
\hline 10 & $1-1$ & {$\left[\begin{array}{cc}1 & -1 \\
-1 & 1\end{array}\right]$} \\
\hline 11 & 11 & {$\left[\begin{array}{cc}1 & 1 \\
1 & 1\end{array}\right]$} \\
\hline
\end{tabular}




\section{The Methodology}

The proposed surveillance module has four phases, they are:

\subsection{Video capture}

A pre-installed Camera captures a surveillance video at any location of a surveillance environment. The selected location has to be proper enlighten and serves security requests. The captured video would be converted into MPEG format if it was not. Conversion allows proposed module to deal with the video.

\subsection{Pre-processing:}

Each captured video would be converted into JPEG format to accelerate processing. Then, JPEG images would be converted to binary images which simulate MMCA based module. Finally, the binary images would be represented in bipolar representation with eliminations of diagonal zeros corresponding to MMCA enhancement.

\subsection{Training phase}

After Pre-processing, the image streams go through the training phase. At the first training cycle, two typical True images (Secured and non-secured images) had been selected for each image stream. Through the later cycles, more true images had been selected to train on. It might be either secured or non-secured images. The number of images had increased till the end of training cycles. These selected images for each training cycle would be used during the training phase to generate weights and lookup table according to the learning phase of MMCA Associative memory. This would be done according to the following proposed Algorithm 3.

Algorithm 3: Training Phase for Multiple Shoot In-Door surveillance Module.

Input: Training images.

Output: Lookup table for one training image.

Step 1: Repeat steps 2 to 6 for each training cycle:

Step 2: Convert the training image to JPG format.

Step 3: Convert the training JPG image to black and white image.

Step 4: Represent the training image in bipolar representation using a hard -limiter function as follows:

$$
P(x, y)=\left\{\begin{array}{cll}
1 & \text { if } & P(x, y)>0 \\
-1 & \text { if } & P(x, y) \leq 0
\end{array}\right.
$$

Step 5: Applying the learning phase of MMCA.

Step 6: Save the resulted learning output in a lookup table Step 7: End.

\subsection{Analyzing phase}

Post-training phase, when the captured video came through the proposed module. It has re-processed in the same way. The production would be an image stream for each video in bipolar representation.

Weight have been obtained from the training phase. For each image stream, a convergence image stream has been generated to be compared with the original image stream that depend on the selected secured training image in the training phase. According to MIMCA Associative Memory Convergence phase, the produced 
convergence image stream allows the proposed module to decide whether the image stream (or captured video) is secured or not secured case. Analyzing process would be implemented according to proposed Algorithm 4.

Algorithm 4: Analysis Phase Algorithm for One Shoot In-Door surveillance Module.

Input: Image stream and resulted lookup table of the training phase.

Output: Secure or unsecured deision.

Step 1: Repeat steps 2 to 6:

Step 2: Convert the image to JPG format.

Step 3: Convert the JPG image to black and white image.

Step 4: Represent the image in bipolar representation using a hard -limiter function as follows:

$$
P(x, y)=\left\{\begin{array}{cll}
1 & \text { if } & P(x, y)>0 \\
-1 & \text { if } & P(x, y) \leq 0
\end{array}\right.
$$

Step 5: Applying the Convergence phase of MMCA Algorithm.

Step 6: Based on the lookup table

If the MMCA converge to the secure training image then

Return Secure.

Else

\section{Return Unsecure}

Step 7: End.

\subsection{Results and Discussion}

To prove the efficiency 11 video were captured as a sample (See Table 1). The results have been gathered. Accuracy has been calculated by proper equation for each training cycle that illustrates proposed module performance which is percentage ratio. It illustrates the relation between true and false cases for each image stream and its corresponding selected training image (See equation 2).

Where:

$$
A=\frac{C_{t}}{T_{n}} \times 100
$$

$\boldsymbol{A}$ is Accuracy ratio.

$\boldsymbol{C}_{\boldsymbol{t}}$ is Case number of true result (Neither Secured or unsecured).

$\boldsymbol{T}_{\boldsymbol{n}}$ is the total number of frames or images.

\begin{tabular}{|c|c|c|c|c|c|}
\hline Video No. & $\begin{array}{l}\text { Duration } \\
\text { (Min:Sec) }\end{array}$ & $\begin{array}{c}\text { Accuracy } \\
\text { at last } \\
\text { training } \\
\text { cycle } \\
\% \\
\end{array}$ & Video No. & $\begin{array}{l}\text { Duration } \\
\text { (Min:Sec) }\end{array}$ & $\begin{array}{c}\text { Accuracy } \\
\text { at last } \\
\text { training } \\
\text { cycle } \\
\%\end{array}$ \\
\hline 01 & 01:16 & $98.9 \%$ & 07 & $00: 38$ & $100 \%$ \\
\hline 02 & 01:25 & $98 \%$ & 08 & 01:00 & $100 \%$ \\
\hline 03 & 01:00 & $100 \%$ & 09 & 02:00 & $100 \%$ \\
\hline 04 & 01:00 & $98.9 \%$ & 10 & 01:03 & $98.9 \%$ \\
\hline 05 & $01: 40$ & $97 \%$ & 11 & $01: 25$ & $100 \%$ \\
\hline 06 & $02: 28$ & 98.9 & & & \\
\hline
\end{tabular}

Table l. Captured videos. 
Appendix 1 shows results for all image streams (or Captured videos) with the accuracy and number of frames. Except for videos (No.5 and No.10), the accuracy was between $(62-76 \%)$ at first training cycle with two typical true images (one secured and the other was not secured). In Video No.5, at the first training cycle low accuracy has been obtained (43\%). There were two reasons behind that. First one was moving objects outside the surveillance location (Moving cars showed from the window). This has a negative effect on the accuracy level (see figure. 5).

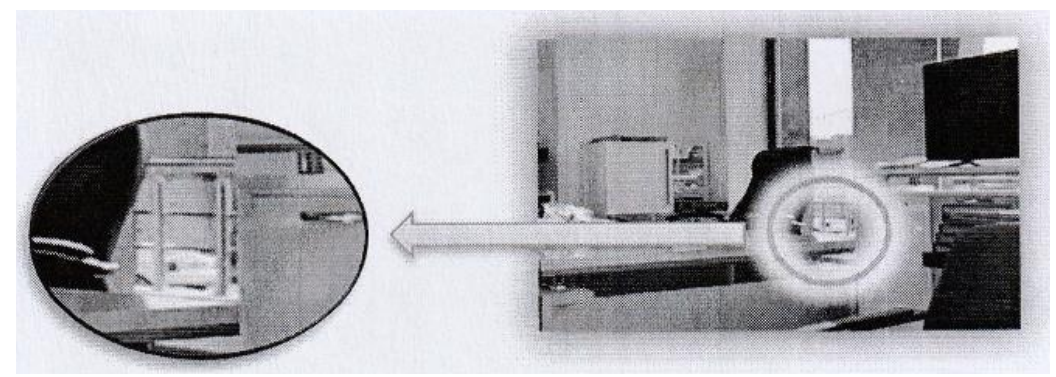

Figure 5. Outside moving objects (A car could be noticed).

The second reason was MMCA correlation error because of the similarity of colors like a light or dark colors of the environment objects and moving objects (a dark color dressed person sat on a dark color chair) as shown in figure 6.

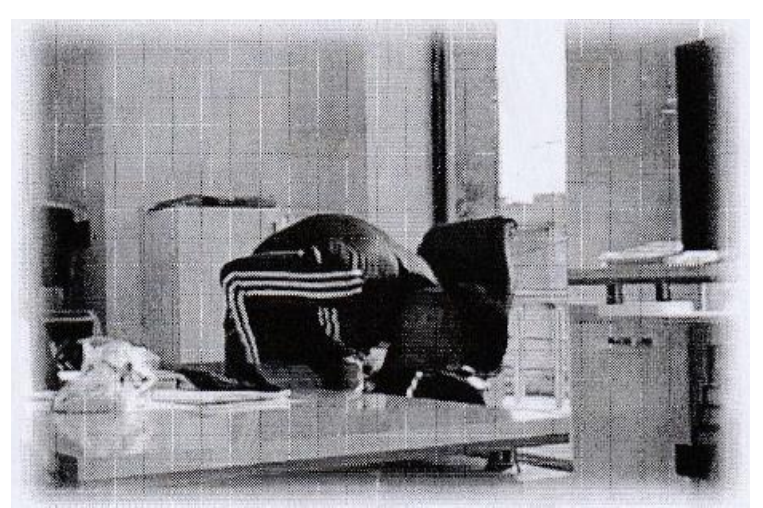

Figure 6. Color Similarity phenomenon.

In Video No.10, at the first training cycle also low accuracy had been obtained (48\%), this came after far distance moving objects (a person standing far away from camera). The far distance moving objects might not be recognized as non-secured case. It considered a false case that harmed accuracy (see figure 7).

At the later training cycle, all captured videos had an incremental ratio of accuracy. The purpose behind that is selecting more true images (Secured and Nonsecured Images).

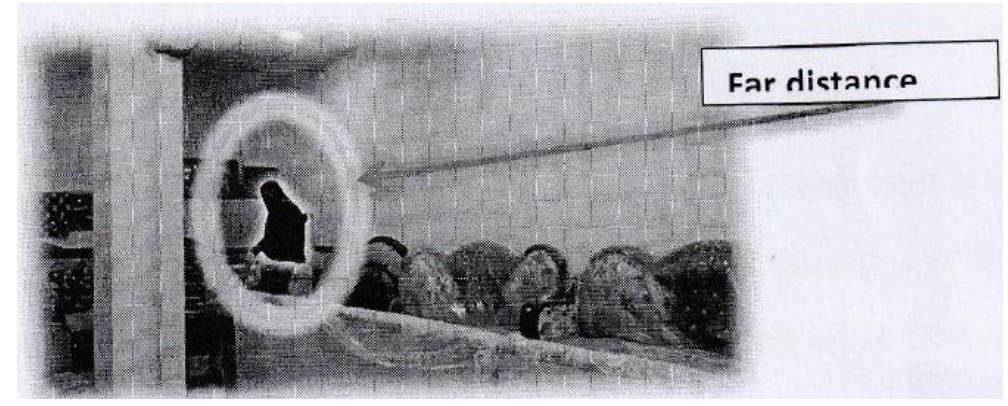

Figure 7. Far distance sensitivity phenomenon. 
This enhanced proposed module performance until the highest accuracy (see appendix 1). Accuracy was between (97-100\%). This means that the additional true case increased accuracy ratio in a good scale. These additional training images give more capable of recognizing true images as illustrate in figure. 8 and 9.

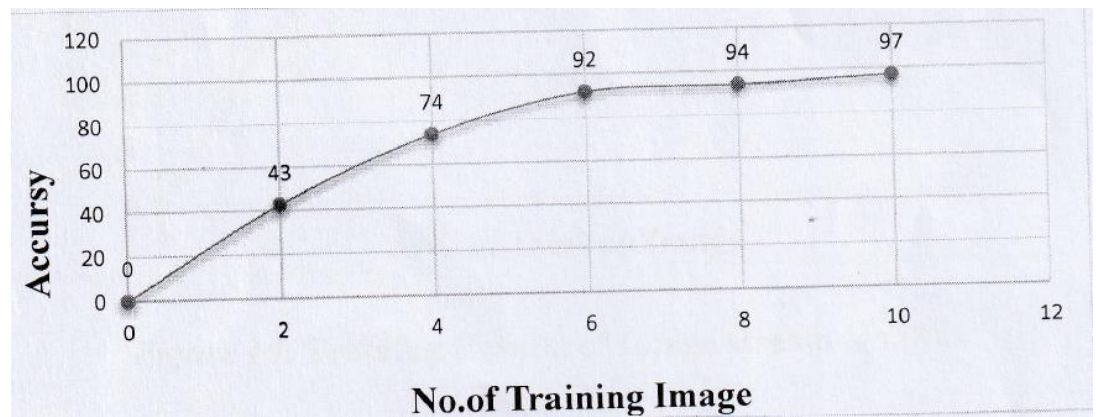

Figure 8. Training Results of Image stream No. 5.

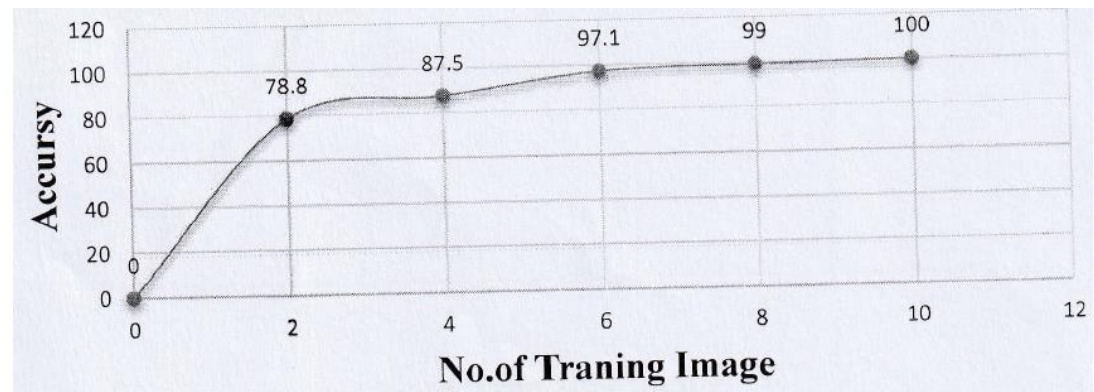

Figure. 9: Training Results of Image stream No. (7).

As mentioned before, the far distance, correlation error, and outside movements had been overcome by adding more true images.

Other videos showed regular results because no effected phenomenon had been noticed at video durations. So, the later training cycle had less benefit but it also increased the accuracy ratio which were between (62.7-78.8\%) at the first training cycle with two training images (secured and non-secured Images). But they were between $(97-100 \%)$ at the fifth cycle with (10) training images. This could be noticed for other videos (see: Appendix 1 and figure 10 and 11 for example).

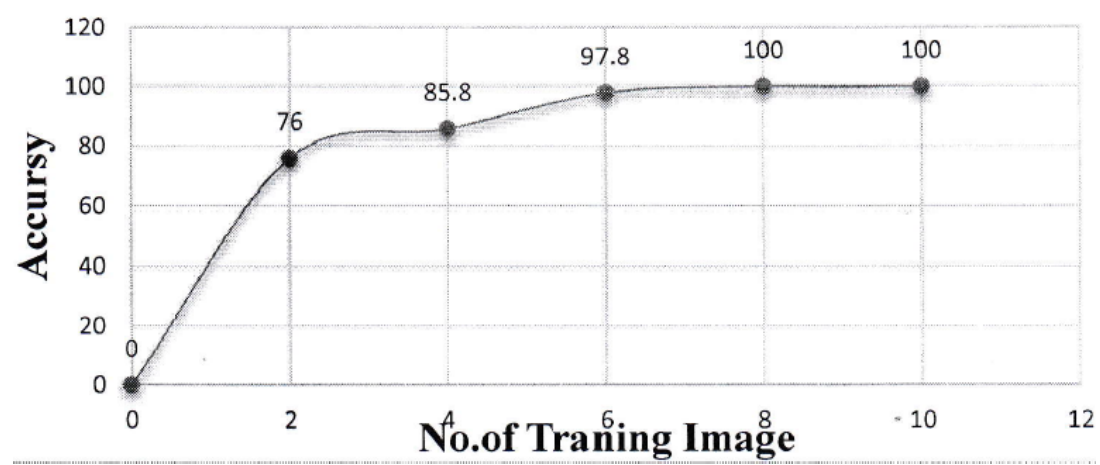

Figure 10: Training Results of Image stream No.(8). 


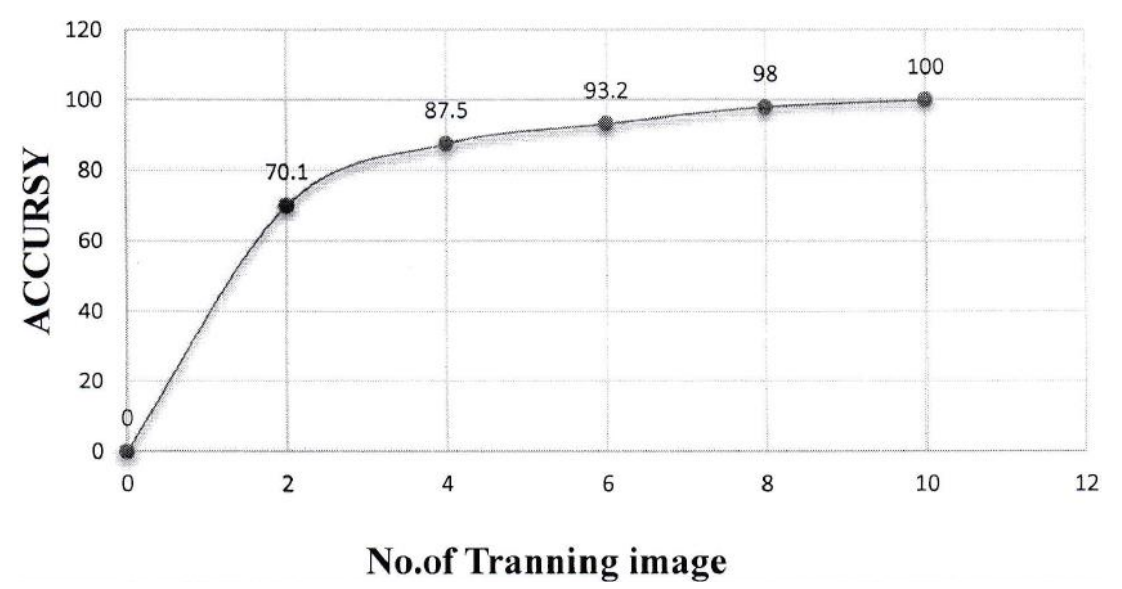

Figure 11: Training Results of Image stream No. (11).

The results show that the accuracy ratio was high (97 to 100\%) that proved the efficiency of the proposed module. The most important issue is using non-traditional techniques in the proposed module. The MMCA associative memory is an efficient artificial intelligent technique that gives an In-Door surveilling module in high performance. Within it has a fixed size of network. It is noticed that it has only two nodes.

The total time of analyzing was between (10.2-23.2 Sec). Time is almost a real time that enables the proposed module to alert the user in true non-secured cases. Which is an advantage of using the artificial intelligence technique.

The proposed module has overcome most security and surveillance problem that might face such a MMCA based module. It met the security request and give a high performance.

\section{Conclusion}

Through reviewing results and discussions, The MMCA Associative Memory is an efficient technique of Artificial intelligence that can be used in In-Door Surveilling. MMCA Associative Memory insufficiency has been overcome by adding more true training images. Far objects or outside objects or their reflections correlation errors has been managed by additional true images either secured or non-secured. This has enhanced the MMCA Associative Memory capabilities.

It is important to mention that selecting camera location has to be in proper location. Because windows or any reflective material have negative effects on current module efficiency. In other way, cameras must be distributed to cover the average distance that allows them to recognize any moving objects. 


\section{REFERENCES}

[1] Nisreen Abd Alhadi Jabr1 and Emad I Abdul Kareem2, "Novel HeteroAssociative Memory: A Modified Bidirectional Associative Memory", 2016.

[2] Akash Kumbharel, Rohit Nayak, et al. (2015), "A dynamic environment monitoring system".

[3] Valery A. Petrushin, Omer Shakil, et al. (2006), "Multiple-sensor Indoor Surveillance System".

[4] Emad Issa Abdul Kareem *1, Aman Jantan *2, 2009, "An approach for monitoring pedestrian by using multi-connect architected MCA associative memory".

[5] Emad I Abdul Kareem, et al, "Multi-Connect Architecture (MCA) Associative Memory: A Modified Hopfield Neural Network", 2012.

[6] Leopord, Hakizimana \& Wilson, Kipruto \& Cheruiyot. (2016). The Use of an Intelligent Surveillance System in Developing Countries-Rwanda. 6.

[7] Rusul Hussein Hasanl, Emad I Abdul Kareem," An Image Processing Oriented Optical Mark Reader Based on Modify Multi - Connect Architecture MMCA", 2015.

[8] Mete Demircigil, et a1, "ON A Model of Associative Memory with Huge Storage Capacit", 2017.

[9] Sarah H. Khaled, Safana H. Abbas, "Braille Character Recognition Using Associative Memories", International Journal of Engineering Research And Advanced Technology (IJERAT), January- 2017 ,Vol. 3, Issue.1.

[10] X. Qi et al., "Content Oriented Surveillance System Based on Information-Centric Network," 2016 IEEE Globecom Workshops (GC Wkshps), Washington, DC, 2016, pp. 1-6. 


\section{Appendix 1}

\begin{tabular}{|c|c|c|c|c|c|c|c|c|c|c|c|c|}
\hline \multirow{3}{*}{$\begin{array}{l}\text { Video } \\
\text { No. }\end{array}$} & \multirow{3}{*}{$\begin{array}{c}\text { Cycle } \\
\text { No. }\end{array}$} & \multirow{3}{*}{ 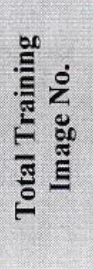 } & \multirow{3}{*}{ 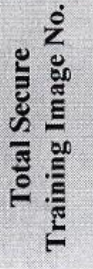 } & \multirow{3}{*}{ 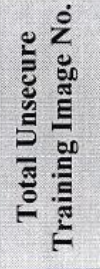 } & \multicolumn{6}{|c|}{ Training Result } & \multirow{3}{*}{$\begin{array}{c}\text { Time } \\
\text { Sec }\end{array}$} & \multirow{3}{*}{$\begin{array}{c}\text { Accuracy } \\
\%\end{array}$} \\
\hline & & & & & \multicolumn{2}{|c|}{ Secure } & \multicolumn{2}{|c|}{ Non-Secure } & \multicolumn{2}{|c|}{ Total Result } & & \\
\hline & & & & & True & False & True & False & True & False & & \\
\hline \multirow{5}{*}{01} & 1 & 2 & 1 & 1 & 17 & 29 & 44 & 1 & 61 & 30 & 2.3 & $67 \%$ \\
\hline & 2 & 4 & 1 & 3 & 19 & 15 & 56 & 1 & 75 & 16 & 5.4 & $82.4 \%$ \\
\hline & 3 & 6 & 1 & 5 & 19 & 3 & 68 & 1 & 87 & 4 & 9.2 & $95.6 \%$ \\
\hline & 4 & 8 & 1 & 7 & 19 & 1 & 70 & 1 & 89 & 2 & 16.7 & $97.8 \%$ \\
\hline & 5 & 10 & 1 & 9 & 19 & 1 & 71 & 0 & 90 & 1 & 22.6 & $98.9 \%$ \\
\hline \multirow{5}{*}{02} & 1 & 2 & 1 & 1 & 63 & 33 & 7 & 0 & 70 & 33 & 3.9 & $67.9 \%$ \\
\hline & 2 & 4 & 1 & 3 & 63 & 10 & 30 & 0 & 93 & 10 & 5.7 & $90 \%$ \\
\hline & 3 & 6 & 1 & 5 & 63 & 6 & 34 & 0 & 97 & 6 & 9 & $94 \%$ \\
\hline & 4 & 8 & 1 & 7 & 63 & 4 & 36 & 0 & 99 & 4 & 16.2 & $96.1 \%$ \\
\hline & 5 & 10 & 3 & 7 & 63 & 2 & 38 & 0 & 101 & 2 & 23.2 & $98 \%$ \\
\hline \multirow{5}{*}{03} & 1 & 2 & 1 & 1 & 61 & 26 & 8 & 0 & 69 & 26 & 2.4 & $72.6 \%$ \\
\hline & 2 & 4 & 1 & 3 & 61 & 2 & 32 & 0 & 93 & 2 & 5.3 & $97.8 \%$ \\
\hline & 3 & 6 & 1 & 5 & 61 & 0 & 34 & 0 & 95 & 0 & 10.2 & $100 \%$ \\
\hline & 4 & 8 & 1 & 7 & 61 & 0 & 34 & 0 & 95 & 0 & 10.2 & $100 \%$ \\
\hline & 5 & 10 & 1 & 9 & 61 & 0 & 34 & 0. & 95 & 0 & 10.2 & $100 \%$ \\
\hline \multirow{5}{*}{04} & 1 & 2 & 1 & 1 & 40 & 22 & 33 & 0 & 73 & 22 & 2.3 & $76.8 \%$ \\
\hline & 2 & 4 & 1 & 3 & 40 & 6 & 49 & 0 & 89 & 6 & 5.5 & $93.1 \%$ \\
\hline & 3 & 6 & 1 & 5 & 40 & 4 & 51 & 0 & 91 & 4 & 9.1 & $95.7 \%$ \\
\hline & 4 & 8 & 1 & 7 & 40 & 2 & 53 & 0 & 93 & 2 & 13.9 & $97.9 \%$ \\
\hline & 5 & 10 & 1 & 9 & 40 & 1 & 54 & 0 & 94 & 1 & 19.9 & $98.9 \%$ \\
\hline \multirow{5}{*}{05} & 1 & 2 & 1 & 1 & 40 & 57 & 3 & 2 & 43 & 57 & 2.5 & $43 \%$ \\
\hline & 2 & 4 & 1 & 3 & 55 & 24 & 19 & 2 & 74 & 26 & 5.6 & $74 \%$ \\
\hline & 3 & 6 & 1 & 5 & 33 & 5 & 59 & 3 & 92 & 8 & 9.9 & $92 \%$ \\
\hline & 4 & 8 & 1 & 7 & 34 & 3 & 60 & 3 & 94 & 6 & 11.1 & $94 \%$ \\
\hline & 5 & 10 & 2 & 8 & 35 & 2 & 62 & 1 & 97 & 3 & 19.3 & $97 \%$ \\
\hline \multirow{5}{*}{06} & 1 & 2 & 1 & 1 & 42 & 22 & 30 & 0 & 72 & 22 & 2.4 & $76.5 \%$ \\
\hline & 2 & 4 & 1 & 3 & 42 & 14 & 39 & 0 & 81 & 14 & 5.2 & $86.1 \%$ \\
\hline & 3 & 6 & 1 & 5 & 42 & 5 & 47 & 0 & 89 & 5 & 9.1 & $94.6 \%$ \\
\hline & 4 & 8 & 1 & 7 & 42 & 3 & 49 & 0 & 91 & 3 & 13.9 & $96.8 \%$ \\
\hline & 5 & 10 & 1 & 9 & 42 & 1 & 51 & 0 & 93 & 1 & 19.9 & $98.9 \%$ \\
\hline
\end{tabular}




\begin{tabular}{|c|c|c|c|c|c|c|c|c|c|c|c|c|}
\hline \multirow{3}{*}{$\begin{array}{l}\text { Video } \\
\text { No. }\end{array}$} & \multirow{3}{*}{\begin{tabular}{|c} 
Cycle \\
No.
\end{tabular}} & \multirow{3}{*}{ 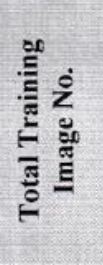 } & \multirow{3}{*}{ 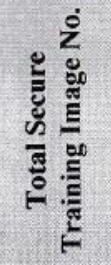 } & \multirow{3}{*}{ 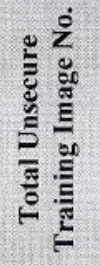 } & \multicolumn{6}{|c|}{ Training Result } & \multirow{3}{*}{$\begin{array}{l}\text { Time } \\
\text { Sec }\end{array}$} & \multirow{3}{*}{$\begin{array}{c}\text { Accuracy } \\
\%\end{array}$} \\
\hline & & & & & \multicolumn{2}{|c|}{ Secure } & \multicolumn{2}{|c|}{ Non-Secure } & \multicolumn{2}{|c|}{ Total Result } & & \\
\hline & & & & & True & False & True & False & True & False & & \\
\hline \multirow{5}{*}{07} & 1 & 2 & 1 & 1 & 46 & 22 & 36 & 0 & 82 & 22 & 2.4 & $78.8 \%$ \\
\hline & 2 & 4 & 1 & 3 & 48 & 13 & 48 & 1 & 96 & 14 & 5.4 & $87.5 \%$ \\
\hline & 3 & 6 & 1 & 5 & 48 & 2 & 53 & 1 & 101 & 3 & 8.9 & $97.1 \%$ \\
\hline & 4 & 8 & 1 & 7 & 49 & 1 & 54 & 0 & 103 & 1 & 13.2 & $99 \%$ \\
\hline & 5 & 10 & 1 & 9 & 50 & 0 & 54 & 0 & 104 & 0 & 19.2 & $100 \%$ \\
\hline \multirow{5}{*}{08} & 1 & 2 & 1 & 1 & 39 & 21 & 31 & 1 & 70 & 22 & 2.4 & $76 \%$ \\
\hline & 2 & 4 & 1 & 3 & 39 & 11 & 40 & 1 & 79 & 12 & 5.4 & $85.5 \%$ \\
\hline & 3 & 6 & 1 & 5 & 39 & 0 & 51 & 2 & 90 & 2 & 9.1 & $97.8 \%$ \\
\hline & 4 & 8 & 1 & 7 & 41 & 0 & 51 & 0 & 92 & 0 & 13.5 & $100 \%$ \\
\hline & 5 & 10 & 3 & 7 & 41 & 0 & 51 & 0 & 92 & 0 & 19 & $100 \%$ \\
\hline \multirow{5}{*}{09} & 1 & 2 & 1 & 1 & 32 & 38 & 32 & 0 & 64 & 38 & 3.9 & $62.7 \%$ \\
\hline & 2 & 4 & 1 & 3 & 32 & 14 & 56 & 0 & 88 & 14 & 5.6 & $86.2 \%$ \\
\hline & 3 & 6 & 1 & 5 & 32 & 8 & 61 & 0 & 93 & 8 & 9.1 & $91.1 \%$ \\
\hline & 4 & 8 & 1 & 7 & 32 & 2 & 68 & 0 & 100 & 2 & \begin{tabular}{|l|}
13.9 \\
\end{tabular} & $98 \%$ \\
\hline & 5 & 10 & 1 & 9 & 32 & 0 & 70 & 0 & 102 & 0 & 18 & $100 \%$ \\
\hline \multirow{5}{*}{10} & 1 & 2 & 1 & 1 & 39 & 49 & 7 & 0 & 46 & 49 & 2.5 & $48.4 \%$ \\
\hline & 2 & 4 & 1 & 3 & 23 & 4 & 52 & 16 & 75 & 20 & 5.4 & $78.9 \%$ \\
\hline & 3 & 6 & 2 & 4 & 40 & 6 & 47 & 2 & 87 & 8 & \begin{tabular}{|l|}
11.3 \\
\end{tabular} & $91.5 \%$ \\
\hline & 4 & 8 & 3 & 5 & 39 & 3 & 53 & 0 & 92 & 3 & 14.1 & $96.8 \%$ \\
\hline & 5 & 10 & 3 & 7 & 41 & 1 & 53 & 0 & 94 & 1 & 20 & $98.9 \%$ \\
\hline \multirow{5}{*}{11} & 1 & 2 & 1 & 1 & 52 & 31 & 21 & 0 & 73 & 31 & 2.3 & $70.1 \%$ \\
\hline & 2 & 4 & 1 & 3 & 52 & 13 & 39 & 0 & 91 & 13 & 5.4 & $87.5 \%$ \\
\hline & 3 & 6 & 1 & 5 & 51 & 6 & 46 & 1 & 97 & 7 & 9 & $93.2 \%$ \\
\hline & 4 & 8 & 1 & 7 & 52 & 1 & 50 & 1 & 102 & 2 & 13.6 & $98 \%$ \\
\hline & 5 & 10 & 2 & 8 & 53 & 0 & 51 & 0 & 104 & 0 & 19 & $100 \%$ \\
\hline
\end{tabular}

